\title{
Numerical Simulation of the Elastic Properties of Rh-20at\%Pd Nanowire
}

\author{
J. Davoodi and M. J. Moradi
}

\begin{abstract}
The aim of this research was to calculate Yong modulus, Bulk modulus and the elastic constants of Rh-20at\%Pd (atom percent) nanowire. The molecular dynamics simulation technique was used to calculate the mechanical properties at constant temperature, constant pressure ensemble. The cohesive energy of the model nanowire systems was calculated by Quantum Sutton-Chen many body potential. The temperature and the pressure of the system were controlled by Nose-Hoover thermostat and Berendsen barostat, respectively. In addition effects of the diameter of nanowire on the mechanical properties were studied. The obtained results show that, when the diameter of Rh-20at\%Pd nanowire increase, elastic constants, bulk modulus and Young modulus all increase, and when the diameter reaches about $5.5 \mathrm{~nm}$, the properties began to level off and remain constant.
\end{abstract}

Index Terms-Mechanical properties, molecular dynamics simulation, Rh-20at\%Pd nanowire.

\section{INTRODUCTION}

The molecular dynamics simulation technique is a very powerful tool for studying of physical properties of materials in macro and nano scale. In this investigation, we used molecular dynamics simulation technique to study of Young modulus, bulk modulus as well as elastic constants of pure $\mathrm{Rh}$ and Pd metals as well as $\mathrm{Rh}-20 \mathrm{at} \% \mathrm{Pd}$ nanowire. Our simulation applied at constant temperature and pressure (NPT) ensemble. The quantum Sutton-Chen (Q-SC) potential [1]-[3] parameters of the pure $\mathrm{Pd}$ and $\mathrm{Rh}$ metals were used as interatomic potential parameters to calculate the cohesive energy as well as the mechanical properties of Pd and $\mathrm{Rh}$ pure metals and $\mathrm{Rh}-20 \mathrm{at} \% \mathrm{Pd}$ random alloys. We have calculated mechanical properties and cohesive energy of Pd, $\mathrm{Rh}$ elements under atmospheric pressure for evaluating computer codes and the interatomic potential.

The Pd-based alloys have been studied for more than 100 years with respect to both fundamental and applied aspects. Especially, in the last three decade thermodynamic and mechanical properties of pd-based alloys were studied by molecular dynamics simulation technique [1], [4], [5]. Among the Pd-based alloys, Rh-Pd alloy is of special interest owing to their increased hydrogen absorption capacity. Moreover, Pd based alloys can potentially offer improvements to pure Pd membranes. Very few experimental

Manuscript received December 19, 2011; revised January 18, 2012.

This work was supported by the University of Zanjan.

J. Davoodi and M. J. Moradi are with the Department of Physics, University of Zanjan, Zanjan, Iran (e-mail: jdavoodir@ znu.ac.ir; mjm.znu64@gmail.com). data are available for the mechanical properties of Rh-Pd nanowire; therefore calculation of thermal and mechanical properties of this nanowire is very important for industrial applications.

This paper is organized as follows. Section 2 details our simulation methodology that we use for equilibrium molecular dynamics simulation. In section 3 , we present simulation results for the materials under investigation.

\section{Simulation Details}

\section{A. Interatomic Potential}

In the present molecular dynamics simulation a Quantum Sutton-Chen [6] many body potential was employed, this potential give very good value for cohesive energy of Rh and Pd atoms [7], [8]. In this potential function, the cohesive energy of system obtain from following equation

$$
\begin{gathered}
U=\sum_{i}\left[\frac{1}{2} \sum_{j \neq i} \varepsilon V\left(r_{i j}\right)-c \varepsilon \sqrt{\rho_{i}}\right] \\
V\left(r_{i j}\right)=\left(\frac{a}{r_{i j}}\right)^{n}, \rho_{i}=\sum_{j \neq i}\left(\frac{a}{r_{i j}}\right)^{m}
\end{gathered}
$$

The first term, $V\left(r_{i j}\right)$, describes the pairwise repulsive interaction between atoms $i$ and $j$, and the second term, $\rho_{i}$, represents the many-body cohesive term associated with atom $i . r_{i j}$ is the distance between atoms $i$ and $j, a$ is a length parameter scaling, $c$ is a dimensionless parameter scaling, $\varepsilon$ sets the overall energy scale, and $n, m$ are integer parameters. The potential parameters of $\mathrm{Rh}, \mathrm{Pd}$ and $\mathrm{Rh}-\mathrm{Pd}$ are listed in Table I. To construct the potential for the binary alloy state, $\mathrm{Rh}-20 \% \mathrm{Pd}$, from the corresponding Q-SC potentials for the elemental state, we used the following mixing rule [7], [9]

$$
\begin{gathered}
m_{i j}=\frac{\left(m_{i}+m_{j}\right)}{2}, n_{i j}=\frac{\left(n_{i}+n_{j}\right)}{2} \\
\varepsilon_{i j}=\left(\varepsilon_{i} \varepsilon_{j}\right)^{1 / 2}, a_{i j}=\left(a_{i} a_{j}\right)^{1 / 2}
\end{gathered}
$$

TABLE I: THE Q-SC POTENTIAL PARAMETER FOR RH-PD MODEL ALLOY SYSTEM

\begin{tabular}{cccccc}
\hline \hline Interaction & $\varepsilon(\mathrm{eV})$ & $a(\AA)$ & $c$ & $n$ & $m$ \\
\hline Rh-Rh & 0.002461 & 3.7984 & 305.49 & 13 & 13 \\
Pd-Pd & 0.003286 & 3.8813 & 148.20 & 12 & 6 \\
Rh-Pd & 0.002843 & 3.8396 & ----- & 12.5 & 9.5
\end{tabular}




\section{B. Temperature and Pressure Control}

The extended system method was used for temperature control. This method was originally introduced by Nose [10] and subsequently developed by Hoover [11], therefore this method was called Nose-Hoover thermostat. The introduction of this mechanism modifies the standard velocity Verlet equations of motion to the following form [12], [13]

$$
\begin{array}{r}
\vec{r}_{i}(t+\delta t)=\vec{r}_{i}(t)+\delta t \overrightarrow{\mathrm{v}}_{i}(t)+\frac{1}{2} \delta t^{2}\left[\frac{\vec{f}_{i}(t)}{m_{i}}-\zeta(t) \overrightarrow{\mathrm{v}}_{i}(t)\right] \\
\zeta\left(t+\frac{1}{2} \delta t\right)=\zeta(t)+\frac{\delta t}{2 Q}\left[\sum_{i}^{N} m_{i} \overrightarrow{\mathrm{v}}_{i}^{2}(t)-g k_{b} T\right] \\
\overrightarrow{\mathrm{v}}_{i}\left(t+\frac{1}{2} \delta t\right)=\overrightarrow{\mathrm{v}}_{i}(t)+\frac{\delta t}{2}\left[\frac{\vec{f}_{i}(t)}{m_{i}}-\zeta(t) \overrightarrow{\mathrm{v}}_{i}(t)\right] \\
\zeta(t+\delta t)=\zeta\left(t+\frac{1}{2} \delta t\right)+\frac{\delta t}{2 Q}\left[\sum_{i}^{N} m_{i} \overrightarrow{\mathrm{v}}_{i}^{2}\left(t+\frac{1}{2} \delta t\right)-g k_{b} T\right] \\
\overrightarrow{\mathrm{v}}_{i}(t+\delta t)=\frac{2}{2+\delta t \zeta(t+\delta t)}\left[\overrightarrow{\mathrm{v}}_{i}\left(t+\frac{1}{2} \delta t\right)+\frac{1}{2} \delta t \frac{\vec{f}_{i}(t+\delta t)}{m_{i}}\right]
\end{array}
$$

where $\zeta$ is the dynamic friction coefficient of the heat bath to which the system is coupled. This coefficient is not a constant and can take on both positive and negative values, leading to the deceleration or acceleration of the atoms respectively, if their total kinetic energy is greater, or smaller, than $g k_{b} T / 2$. A particular parameterization of $Q$ is given by

$$
Q=g k_{b} T \tau^{2}
$$

where $\tau$ is the relaxation time of the thermostat. This parameter controls the speed with which the thermostat damps down the fluctuations in the temperature. The number of degrees of freedom, $g$, is given by $g=3(N-1)$.

The Berendsen barostat [14] was used to pressure control of system. This barostat uses a scale factor, $\mu$, which is a function of instantaneous pressure, $P$, to scale lengths in the system.

$$
\begin{aligned}
& x(i) \rightarrow \mu x(i) \\
& y(i) \rightarrow \mu y(i) \\
& z(i) \rightarrow \mu z(i) \\
& L \rightarrow \mu L
\end{aligned}
$$

where $\mu$ is given by

$$
\mu=\left[1-\frac{\delta t}{\tau_{p}}\left(P-P_{0}\right)\right]^{1 / 3}
$$

Here, $\tau_{p}$ is the rise time of the barostat, and $P_{0}$ is the set point pressure. The system pressure is set toward a desired value by changing the dimensions of the simulation cell size during the simulation.

\section{Mechanical Properties}

The quantities which are calculated in this molecular dynamics simulation were the Young modulus, the elastic stiffness constants as well as the bulk modulus. According to the Hooke's law, for small deformation, the stress components $\sigma_{i j}$ are directly proportional to the strain components $\varepsilon_{i j}$. This relation can be expressed in mathematical terms as

$$
\sigma_{i j}=C_{i j k l} \varepsilon_{k l}
$$

where $C_{i j k l}$ are the elastic stiffness constants. The number of independent elastic stiffness constants is reduced if the crystal possesses symmetry elements. The elastic constants reduce to three constants, $C_{11}, C_{12}$ and $C_{44}$ for the cubic crystal by reason of symmetry [15]. In terms of interatomic potential, these quantities can be expressed as [16]

$$
C_{11}=\frac{1}{\Omega} \frac{\partial^{2} U}{\partial \varepsilon_{11}^{2}}, C_{12}=\frac{1}{\Omega} \frac{\partial^{2} U}{\partial \varepsilon_{11} \partial \varepsilon_{22}}, C_{44}=\frac{1}{4 \Omega} \frac{\partial^{2} U}{\partial \varepsilon_{12}^{2}}
$$

where $\varepsilon_{11}, \varepsilon_{12}$ and $\varepsilon_{22}$ are the components of strain tensor, $\Omega$ is the volume, and $U$ is the energy is given by Eq. 1, per atom.

The bulk modulus, which measure how the volume of the solid change with hydrostatic pressure, denoted by $B$. this is related to interatomic potential via [16]

$$
B=\Omega\left(\frac{\partial^{2} U}{\partial \Omega^{2}}\right)_{\Omega=\Omega_{0}}
$$

where $\Omega_{0}$ is the equilibrium value of $\Omega$.

We have used the Reuss assumption to estimate the Young modulus $(E)$ according the following relations [17]

$$
E=9 G B /(3 B+G)
$$

where $G$ is the shear modulus.

$$
G=5\left(C_{11}-C_{12}\right) /\left(4 C_{44}+3\left(C_{11}-C_{12}\right)\right)
$$

\section{Simulation Data}

In this research, the molecular dynamics simulation is performed for different size of $\mathrm{Rh}-20 \mathrm{at} \% \mathrm{Pd}$ nanowire in NPT ensemble. We changed the diameter of nanowire and calculated mechanical properties, therefore size dependent of mechanical properties of nanowire were obtained. The simulation time step $(\delta t)$ was set 0.05 fs for every run of MD simulation. The nanowire equilibrated during 10000 time steps in the $\mathrm{T}=100 \mathrm{~K}$. Fig. 1 shows the variation of the energy of pure $\mathrm{Rh}, \mathrm{Pd}$ and $\mathrm{Rh}-20 \mathrm{at} \% \mathrm{Pd}$ alloy in order to detect of the equilibrium state. Moreover variation of the pressure and volume are plotted in the Fig. 2. Both figures show fluctuation around constant value in the energy, pressure as well as volume. For translation from macroscale to nanoscale, the periodic boundary condition applied in the axis of nanowire only and removes at the other directions. 


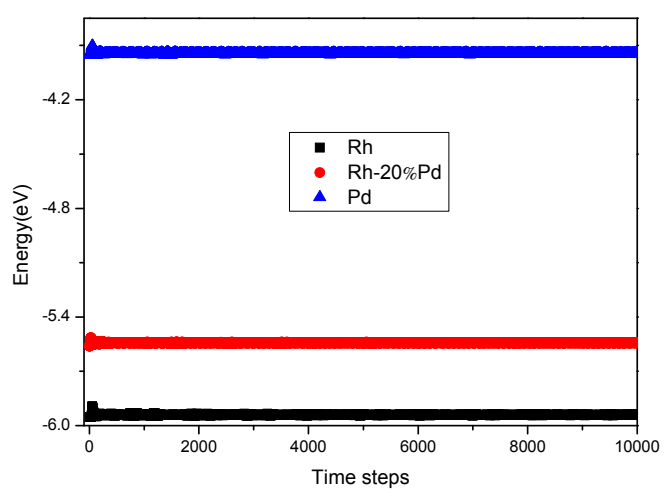

Fig. 1. Time variation of the energy for $\mathrm{Rh}, \mathrm{Rh}-20 \mathrm{at} \% \mathrm{Pd}$ and $\mathrm{Pd}$ nanowires during equilibration.

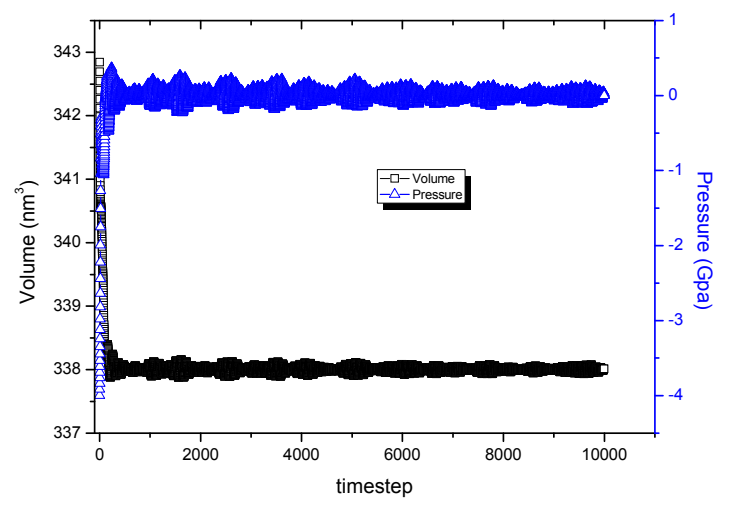

Fig. 2. Time variation of the volume and pressure for the Rh- $20 \mathrm{at} \% \mathrm{Pd}$ nanowire during equilibration.

\section{RESULtS}

At first, the cohesive energy, the elastic constants, the Bulk modulus and the Young modulus were calculated for pure $\mathrm{Rh}$ as well as Pd metals. Our motivation for computing these properties for the elemental materials was to obtain an estimate of the accuracy of the Q-SC potentials. A comparison of the results for the elemental materials shows that our computed values are in reasonable agreement with the experimental data that are available for these materials. This can give an indication of the quality of the alloy potential energy functions that we have constructed on the basis of these elemental potentials. The percentage errors associated with the computed values, as compared to the experimental data are listed in Table II.

The variations of the Young modulus, bulk modulus as well as elastic constants with $\mathrm{Rh}-20 \mathrm{at} \% \mathrm{Pd}$ nanowire diameter were computed in the MD simulation by changing the number of atoms in the nanowire (figures 3-7). Our computed results are in reasonable agreement with other results in the literature where they are available. From these figures we see that there is an initial change in the mechanical properties with the diameter of the nanowire, following which the properties remain unchanged as the nanowire grows in diameter. This is a typical behavior of nanoscopic system in which the mechanical properties, for example, change with size, and then reach a constant value. These results show that, when the diameter of nanowire increase,
$C_{11}, C_{12}, C_{44}$, bulk modulus and Young modulus all increase, and when the diameter reaches about $5.5 \mathrm{~nm}$, the properties began to level off and remain constant.

TABLE II: THE PERCENTAGE ERror OF THE COMPUTEd VALUES

\begin{tabular}{ccc}
\multicolumn{2}{c}{ VIS-À-VIS THE EXPERIMENTAL DATA } \\
\hline \hline Physical properties & $\mathrm{Pd}$ & $\mathrm{Rh}$ \\
\hline$U$ & 0.2 & 0.8 \\
$C_{11}$ & 2.6 & 3.2 \\
$C_{12}$ & 16.2 & 5.7 \\
$C_{44}$ & 44.3 & 17.5 \\
$B$ & 15.7 & 9.5 \\
$E$ & 5.3 & 11.5 \\
\hline \hline
\end{tabular}

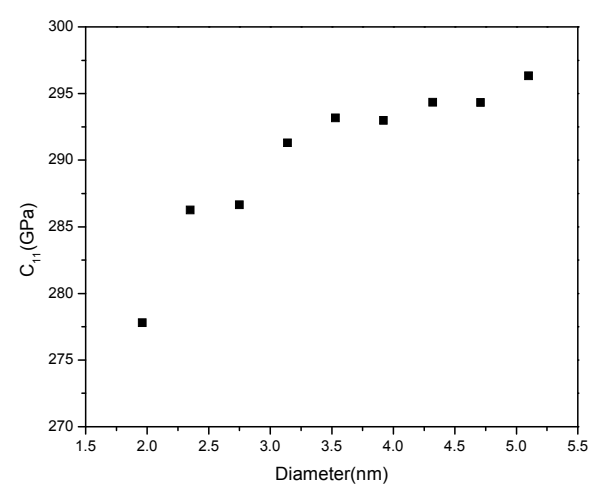

Fig. 3. Variation of elastic constant $\left(C_{11}\right)$ of $\mathrm{Rh}-20 \mathrm{at} \% \mathrm{Pd}$ nanowire with diameter of nanowire.

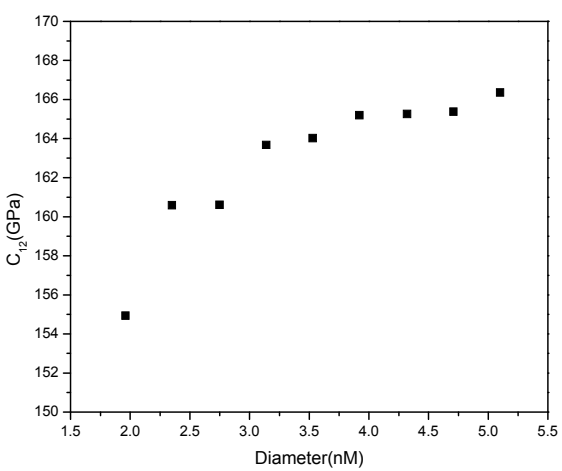

Fig. 4. Variation of elastic constant $\left(C_{12}\right)$ of $\mathrm{Rh}-20 \mathrm{at} \% \mathrm{Pd}$ nanowire with diameter of nanowire.

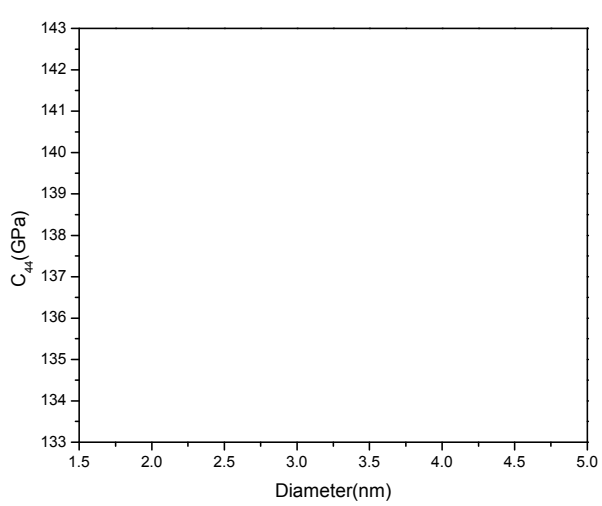

Fig. 5. Variation of elastic constant $\left(C_{44}\right)$ of $\mathrm{Rh}-20 \mathrm{at} \% \mathrm{Pd}$ nanowire with diameter of nanowire. 


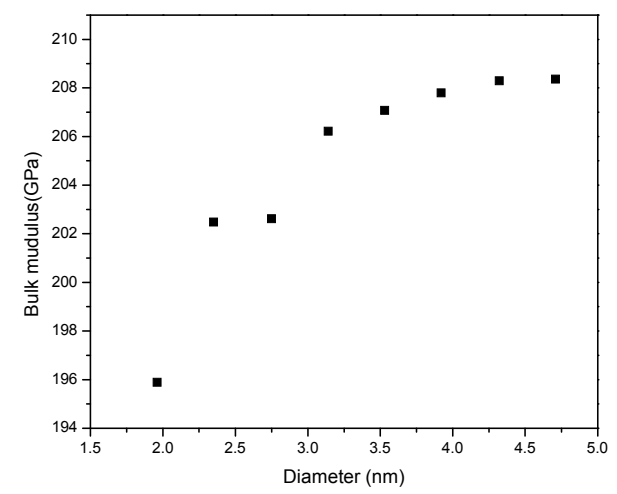

Fig. 6. Variation of bulk modulus of Rh-20at\%Pd nanowire with diameter of nanowire.

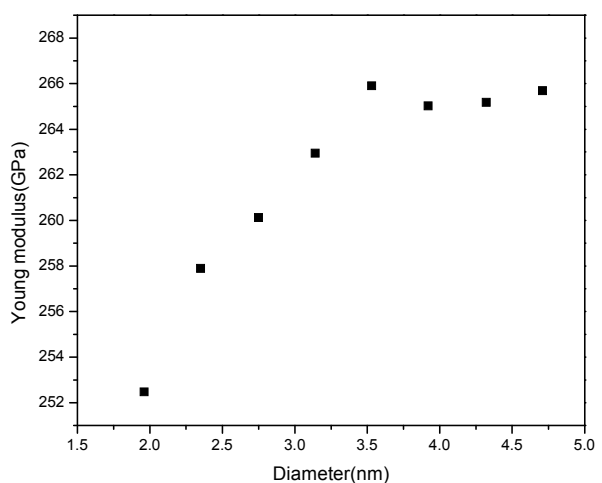

Fig. 7. Variation of Young modulus of Rh-20at\%Pd nanowire with diameter of nanowire.

In summary, the results of molecular dynamics simulation of pure $\mathrm{Rh}$ and $\mathrm{Pd}$ metals show that our simulation results are in reasonable agreement with the experimental data. Moreover, Our MD results of Rh-20at\%Pd nanowire show that, the mechanical properties depend on the diameter of the nanowire and the values of elastic constants, Young modulus and bulk modulus of the nanowire are different from the properties of the bulk alloy.

\section{REFERENCES}

[1] H. H. Kart, M. Tomak, M. Uludogan, and T. Cagin, "Thermodynamical and mechanical properties of Pd-Ag alloys," Comput. Mater. Sci. 32, pp. 107-117, Jan. 2005

[2] Y. Qi, T. Cagın, Y. Kimura, and W.A. Goddard, "Molecular dynamics simulations of glass formation and crystallization in binary liquid metals: Cu-Ag and Cu-Ni," Phys. Rev. B 59, pp. 3527-3533, Sep. 1999.
[3] H. H. Kart, M. Uludogan, T. Cagin, and M. Tomak, "Simulation of crystallization and glass formation of Pd-Ag metal alloys," J. Noncryst. Sol. 342, pp. 6-11, Aug. 2004.

[4] H. H. Kart, M. Tomak, M. Uludogan, and T. Cagin, "Structural and dynamical properties of liquid Pd-Ag alloys," International journal of modern physics B. 18, p. 2257-2269, 2004.

[5] S. O. Kart, M. Tomak, M. Uludogan, and T. Cagin, "Liquid properties of Pd-Ni alloys," J. Noncryst. Sol. 337, pp. 101-108, 2004.

[6] A. P. Sutton and J. Chen, "Long-range Finnis-Sinclair potential," Philosophical Magazine Letters 61, pp. 139-146, March 1990

[7] J. Davoodi and M. Ahmadi, "Thermal properties of $\mathrm{Cu}-\mathrm{Pd}$ alloy under high pressure," Computational Materials Science 49, pp. 21-24, April 2010.

[8] J. Davoodi and J. Moradi, "Temperature effect on the mechanical properties of $\mathrm{Pd}_{3} \mathrm{Rh}$ and $\mathrm{PdRh}_{3}$ ordered alloys," World Academy of Science, Engineering and Technology 78, pp. 354-358, July 2011.

[9] J. C. Barton, J. A. S. Green, and F. A. Lewis, "Changes of electrode potential and electrical resistance as a function of the hydrogen content of some $\mathrm{Pd}+\mathrm{Ni}$ and $\mathrm{Pd}+\mathrm{Rh}$ alloys," Trans. Faraday Soc. 62, pp.960-970, 1966.

[10] S. Nose, "A unified formulation of the constant temperature molecular dynamics methods," J. Chem. Phys. 81, pp. 511-519, Jul. 1984.

[11] W. G. Hoover, "Canonical dynamics: Equilibrium phase-space distribution,” Phys. Rev. A 31, pp. 1695-1697, March 1985.

[12] D. Frenkel and B. Smit, "Understanding molecular simulation from algorithms to application," Academic press, New York, pp. 69-77, 2002.

[13] A. P. Sutton, J. B. Ptthica, H. Rafii-Tabar, J. A. Nieminen, in d. G. Pettifor, A.H. "Cottrell (Eds.), electron theory in alloy design," Institute of Materials, London, 191, 1994.

[14] H. J. C. Berendsen, J. P. M. Postma, W. F. van Gunsteren, A. DiNola, and J. R. Haak, "Molecular dynamics with coupling to an external bath," J. Chem. Phys. 81, pp. 3684-3690, 1984.

[15] Kittel, "Introduction to solid state physics," eighth ed., John Wiley \& Sons, New York, pp. 73-80, 2005.

[16] H. Rafii-Tabar, and A. P. Sutton, "Long-range Finnis-Sinclair potentials for fcc metallic alloys," Philos. Mag. Lett. 63, pp. 217-224, Jan. 1991.

[17] Y. Medkour, A. Roumili, D. Maouche, M. Reffas, and A. Saoudi, "First principles study of structural, elastic and electronic properties of $\mathrm{ACY}_{3}$ $(\mathrm{A}=\mathrm{Al}$, In and $\mathrm{Tl}), "$ Computational materials science, Vol. 47, pp. 973-976, Feb. 2010.

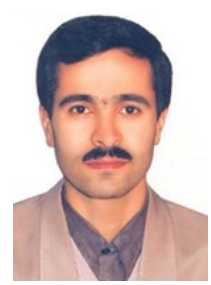

Jamal Davoodi was born on December 1, 1969 in the city of Zanjan, Iran. He got the PHD degree in Isfahan University, Isfahan, Iran, and master degree in Institute for advanceed studies in Basic Science, Zanjan, Iran, and bachelor in Tabriz University, Tabriz, Iran. He is an Associate Professor of physics at Zanjan University, Iran. His research interests include fundamental studies of thermal and mechanical properties of nanostructures using molecular dynamics simulation technique, and MD-based numerical simulation of phase transition (melting \& crystallization) of alloys. 